\title{
Modelling of transient heat transport in metal films using the interval lattice Boltzmann method
}

\author{
A. PIASECKA BELKHAYAT* and A. KORCZAK \\ Institute of Computational Mechanics and Engineering, Silesian University of Technology, 18A Konarskiego St., 44-100 Gliwice, Poland
}

\begin{abstract}
In the paper a description of heat transfer in one-dimensional crystalline solids is presented. The lattice Boltzmann method based on Boltzmann transport equation is used to simulate the nanoscale heat transport in thin metal films. The coupled lattice Boltzmann equations for electrons and phonons are applied to analyze the heating process of thin metal films via laser pulse. Such approach in which the parameters appearing in the problem analyzed are treated as constant values is widely used, but in the paper the interval values of relaxation times and electron-phonon coupling factor are taken into account. The problem formulated has been solved by means of the interval lattice Boltzmann method using the rules of directed interval arithmetic. In the final part of the paper the results of numerical computations are shown.
\end{abstract}

Key words: interval Boltzmann transport equation, interval lattice Boltzmann method, directed interval arithmetic.

\section{Introduction}

In metals the heat transport is mainly realized by two kinds of heat carriers: electrons and quanta of lattice vibrations called phonons [1]. It should be pointed out, that in metals the free electron mechanism of heat transport is much more efficient than the phonon one, because electrons have higher velocities and phonons are more easily scattered than free electrons. These carriers always "move" from the part with the higher temperature to the part with the lower temperature. During this move electrons and phonons carry energy. This kind of phenomena can be described by the Boltzmann transport equation (BTE) [2-5] but the other approaches can be also applied, e.g. [6-8]. The BTE is difficult to solve and for this reason in numerical computations the lattice Boltzmann method (LBM) is used [9-11]. The LBM solves a discretized set of the Boltzmann transport equations. In the paper the coupled lattice Boltzmann equations for electrons and phonons have been assumed. The coupled model contains two energy equations determining the heat exchange in the electron gas and the metal lattice $[12,13]$.

Such approach in which the parameters appearing in the mathematical model are treated as constant values is widely used [14]. In papers [15-17] the mathematical description of heat transfer in $1 \mathrm{D}$ and $2 \mathrm{D}$ silicon film with interval values of phonon relaxation time and boundary conditions has been presented. It should be pointed out that in semiconductors only phonons take part in heat conduction but analyzing this phenomena in metals we should take into account both carriers: phonons and electrons.

In the paper the interval values of relaxation times and electron-phonon coupling factor are taken into account. The relaxation times are estimated experimentally and their actual values

*e-mail: alicja.piasecka@polsl.pl are still a subject of discussion [18]. The analogical problem is with the electron-phonon coupling factor. In the literature we can find a wide range of this value [19]. So it seems natural to take the interval values of relaxation times and coupling factor and this assumption is closer to the real physical conditions of the process analyzed.

In the article the authors present an innovative approach of the described problem using the interval lattice Boltzmann method. Using interval numbers, the mathematical model reflects better the course of the heat flow. There was no such an approach in available literature until now. Moreover, there isn't any known commercial software using the interval LBM, although the presented method can easily be programmed.

Here, the interval lattice Boltzmann method (ILBM) is presented with the approach of the directed interval arithmetic [16, $20,21]$. In this arithmetic a set of proper intervals is extended by improper intervals, and all arithmetic operations and functions are also extended. The main advantage of the directed interval arithmetic $[20,22]$ upon the usual interval arithmetic [23] is that the obtained temperature intervals are much narrower and their width does not increase in time.

In theory as well as in practice it is valuable to develop the interval version of the LBM.

\section{Directed interval arithmetic}

Let us consider a directed interval $\bar{a}$ which can be defined as a set $\mathbf{D}$ of all directed pairs of real numbers defined as follows $[17,20,24]$

$$
\bar{a}=\left[a^{-}, a^{+}\right]:=\left\{\bar{a} \in \mathbf{D} \mid a^{-}, a^{+} \in \mathbf{R}\right\},
$$

where $a^{-}$and $a^{+}$denote the beginning and the end of the interval, respectively. 
The left or the right endpoint of the interval $\bar{a}$ can be denoted as $a^{s}, s \in\{+,-\}$, where $s$ is a binary variable. This variable can be expressed as a product of two binary variables and is defined as

$$
\begin{aligned}
& ++=--=+ \\
& +-=-+=-
\end{aligned}
$$

An interval is called proper if $a^{-} \leq a^{+}$, improper if $a^{-} \geq a^{+}$ and degenerate if $a^{-}=a^{+}$. The set of all directed interval numbers can be written as $\mathbf{D}=\mathbf{P} \cup \mathbf{I}$, where $\mathbf{P}$ denotes a set of all directed proper intervals and I denotes a set of all improper intervals.

Additionally a subset $\mathbf{Z}=\mathbf{Z}_{\mathbf{P}} \cup \mathbf{Z}_{\mathbf{I}} \in \mathbf{D}$ should be defined, where

$$
\begin{aligned}
& \mathbf{Z}_{\mathbf{P}}=\left\{\bar{a} \in \mathbf{P} \mid a^{-} \leq 0 \leq a^{+}\right\} \\
& \mathbf{Z}_{\mathbf{I}}=\left\{\bar{a} \in \mathbf{I} \mid a^{+} \leq 0 \leq a^{-}\right\}
\end{aligned} .
$$

For directed interval numbers two binary variables are defined. The first of them is the direction variable

$$
\tau(\bar{a})=\left\{\begin{array}{lll}
+, & \text { if } a^{-} \leq a^{+} \\
-, & \text {if } a^{-}>a^{+}
\end{array}\right.
$$

and the other is the sign variable

$$
\sigma(\bar{a})=\left\{\begin{array}{ll}
+, & \text { if } a^{-}>0, a^{+}>0 \\
-, & \text { if } a^{-}<0, a^{+}<0
\end{array}, \quad \bar{a} \in \mathbf{D} \backslash \mathbf{Z}\right.
$$

For zero argument $\sigma([0,0])=\sigma(0)=+$, for all intervals including the zero element $\bar{a} \in \mathbf{Z}, \sigma(\bar{a})$ is not defined.

The sum of two directed intervals $\bar{a}=\left[a^{-}, a^{+}\right]$and $\bar{b}=\left[b^{-}, b^{+}\right]$ can be written as

$$
\begin{gathered}
\bar{a}+\bar{b}=\left[a^{-}+b^{-}, a^{+}+b^{+}\right], \\
\bar{a}, \bar{b} \in \mathbf{D} .
\end{gathered}
$$

The difference is of the form

$$
\begin{gathered}
\bar{a}-\bar{b}=\left[a^{-}-b^{+}, a^{+}-b^{-}\right], \\
\bar{a}, \bar{b} \in \mathbf{D} .
\end{gathered}
$$

The quotient of two directed intervals can be written as follows

$$
\bar{a} / \bar{b}=\left\{\begin{array}{r}
{\left[a^{-\sigma(\bar{b})} / b^{\sigma(\bar{a})}, a^{\sigma(\bar{b})} / b^{-\sigma(\bar{a})}\right], \quad \bar{a}, \bar{b} \in \mathbf{D} \backslash \mathbf{Z}} \\
{\left[a^{-\sigma(\bar{b})} / b^{-\sigma(\bar{b}) \tau(\bar{a})}, a^{\sigma(\bar{b})} / b^{-\sigma(\bar{b}) \tau(\bar{a})}\right],} \\
\bar{a} \in \mathbf{Z}, \bar{b} \in \mathbf{D} \backslash \mathbf{Z}
\end{array} .\right.
$$

The product of the directed intervals is described by the following formula

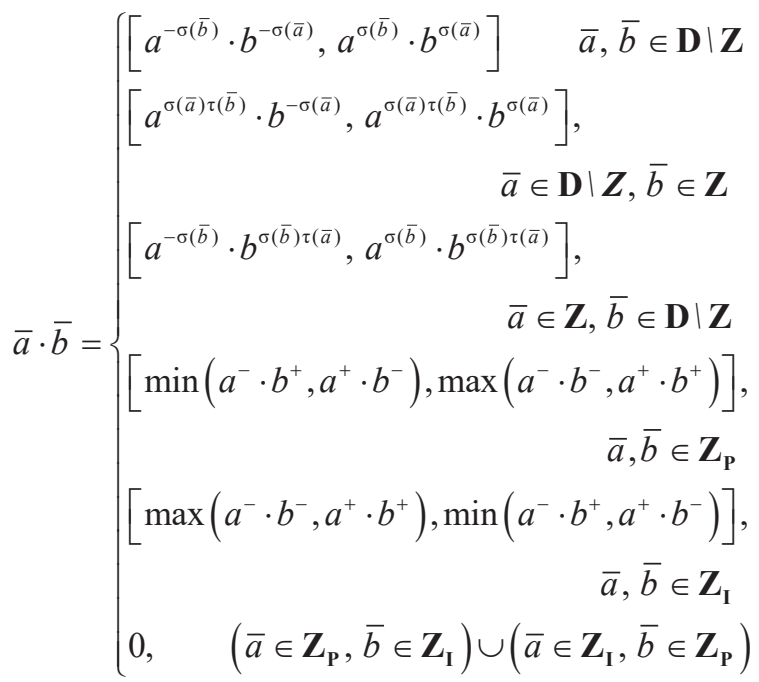

In the directed interval arithmetic two extra operators are defined, inversion of summation

$$
{ }_{\mathbf{D}} \bar{a}=\left[-a^{-},-a^{+}\right], \bar{a} \in \mathbf{D}
$$

and inversion of multiplication

$$
1 / \mathbf{D} \bar{a}=\left[1 / a^{-}, 1 / a^{+}\right], \bar{a} \in \mathbf{D} \backslash \mathbf{Z} .
$$

So, two additional mathematical operations can be defined as follows

$$
\bar{a}{ }_{\mathbf{D}} \bar{b}=\left[a^{-}-b^{-}, a^{+}-b^{+}\right], \bar{a}, \bar{b} \in \mathbf{D}
$$

and

$$
\bar{a} /_{\mathbf{D}} \bar{b}=\left\{\begin{array}{r}
{\left[a^{-\sigma(\bar{b})} / b^{-\sigma(\bar{a})}, a^{\sigma(\bar{b})} / b^{\sigma(\bar{a})}\right],} \\
\bar{a}, \bar{b} \in \mathbf{D} \backslash \mathbf{Z} \\
{\left[a^{-\sigma(\bar{b})} / b^{\sigma(\bar{b})}, a^{\sigma(\bar{b})} / b^{\sigma(\bar{b})}\right],} \\
\bar{a} \in \mathbf{Z}, \bar{b} \in \mathbf{D} \backslash \mathbf{Z} .
\end{array}\right.
$$

Now, it is possible to obtain the number zero by subtraction of two identical intervals $\bar{a}-{ }_{\mathbf{D}} \bar{a}=0$ and the number one as the result of the division $\bar{a}-{ }_{\mathbf{D}} \bar{a}=1$, which was impossible when applying classical interval arithmetic [25].

\section{Formulation of the problem before styling}

During the heating of thin metal films via laser pulse the electrons are energized and they subsequently transfer the energy to phonons via coupling between them. The Boltzmann transport equations for the coupled model (1D problem) with two kinds of carriers: electrons (e) and phonons (ph) can be written in the following form [26] 


$$
\begin{gathered}
\frac{\partial f_{e}}{\partial t}+\mathbf{v}_{e} \cdot \nabla f_{e}=\frac{f_{e}^{0}-f_{e}}{\tau_{e}}+g_{e}, \\
\frac{\partial f_{p h}}{\partial t}+\mathbf{v}_{p h} \cdot \nabla f_{p h}=\frac{f_{p h}^{0}-f_{p h}}{\tau_{p h}}+g_{p h},
\end{gathered}
$$

where $f_{e}, f_{p h}$ are the carrier distribution functions, $f_{e}^{0}, f_{p h}^{0}$ are the equilibrium distribution functions given by the Bose-Einstein statistic for phonons [27] and Fermi-Dirac statistic for electrons, $\mathbf{v}_{e}, \mathbf{v}_{p h}$ are the frequency-dependent carrier propagation speeds, $\tau_{e}, \tau_{p h}$ are the frequency-dependent carrier relaxation times and $g_{e}, g_{p h}$ are the carrier generation rates due to external sources such as laser heating.

The BTEs (14) and (15) can be transformed into an equivalent carrier energy density equations [25]

$$
\begin{gathered}
\frac{\partial e_{e}}{\partial t}+\mathbf{v}_{e} \cdot \nabla e_{e}=\frac{e_{e}^{0}-e_{e}}{\tau_{e}}+Q_{e}, \\
\frac{\partial e_{p h}}{\partial t}+\mathbf{v}_{p h} \cdot \nabla e_{p h}=\frac{e_{p h}^{0}-e_{p h}}{\tau_{p h}}+Q_{p h},
\end{gathered}
$$

where $e_{e}, e_{p h}$ are the carrier energy densities, $e_{e}^{0}, e_{p h}^{0}$ are the equilibrium carrier energy densities and $Q_{e}, Q_{p h}^{0}$ are the carrier energy sources related to an unit of volume. The equations (16) and (17) must be supplemented by the adequate boundary-initial conditions.

The electron and phonon energy densities at their equivalent nonequilibrium temperatures are given by the formulas

$$
\begin{gathered}
e_{e}\left(T_{e}\right)=\left(n_{e} \frac{\pi^{2}}{2} \frac{k_{b}^{2}}{\varepsilon_{F}}\right) T_{e}^{2}, \\
e_{p h}\left(T_{p h}\right)=\left(\frac{9 \eta k_{b}}{\Theta_{D}^{3}} \int_{0}^{\Theta_{D} / T_{p h}} \frac{z^{3}}{\exp (z)-1} \mathrm{~d} z\right) T_{p h}{ }^{4},
\end{gathered}
$$

where $T_{e}, T_{p h}$ are the carrier temperatures, $k_{b}$ is the Boltzmann constant, $\varepsilon_{F}$ is the Fermi energy, $n_{e}$ is the electron density, $\Theta_{D}$ is the Debye temperature of the solid, $\eta$ is the number density of oscillators [28].

The electron and phonon energy sources are calculated using the following expressions [26]

$$
\begin{gathered}
Q_{e}=Q^{\prime}-G\left(T_{e}-T_{p h}\right), \\
Q_{p h}=G\left(T_{e}-T_{p h}\right),
\end{gathered}
$$

where $Q^{\prime}$ is the power density deposited by the external source function associated with the laser irradiation [29-31] and $G$ is the electron-phonon coupling factor which characterizes the energy exchange between electrons and phonons $[12,19]$.

\section{Interval lattice Boltzmann method}

The lattice Boltzmann method is a discrete representation of the Boltzmann transport equations. This method is employed to transport modeling of heat carriers. The interval form of the BTEs for 1D problems can be expressed as [28]

$$
\begin{gathered}
\frac{\partial \bar{e}_{e}}{\partial t}+\mathbf{v}_{e} \cdot \nabla \bar{e}_{e}=\frac{\bar{e}_{e}^{0}-\bar{e}_{e}}{\bar{\tau}_{e}}+\bar{Q}_{e}, \\
\frac{\partial \bar{e}_{p h}}{\partial t}+\mathbf{v}_{p h} \cdot \nabla \bar{e}_{p h}=\frac{\bar{e}_{p h}{ }^{0}-\bar{e}_{p h}}{\bar{\tau}_{p h}}+\bar{Q}_{p h},
\end{gathered}
$$

where $\bar{e}_{e}, \bar{e}_{p h}$ are the interval values of carrier energy densities for electrons and phonons respectively, $\bar{e}_{e}^{0}, \bar{e}_{p h}^{0}$ are the interval equilibrium carrier energy densities and $\bar{\tau}_{e}, \bar{\tau}_{p h}$ are the interval relaxation times.

The interval values of the electron and phonon energy sources are calculated using the following formulas according to the rules of directed interval arithmetic [20]

$$
\begin{gathered}
\bar{Q}_{e}=Q^{\prime}-\bar{G}\left(\bar{T}_{e}-\bar{T}_{p h}\right), \\
\bar{Q}_{p h}=\bar{G}\left(\bar{T}_{e}-\bar{T}_{p h}\right),
\end{gathered}
$$

where $\bar{G}$ is the interval value of the electron-phonon coupling factor.

For example, for the interval values of the electron-phonon coupling factor $\bar{G}=\left[2 \times 10^{16}, 2.5 \times 10^{16}\right] \mathrm{W} / \mathrm{m}^{3} \mathrm{~K}$ and the temperatures of electrons $\bar{T}_{e}=[1175,1179.6] \mathrm{K}$ an phonons $\bar{T}_{p h}=[360.2,373.7] \mathrm{K}$, the phonon energy source is calculated using the rules of directed interval arithmetic according to the following formula (see (12))

$$
\begin{aligned}
\bar{Q}_{p h}= & \bar{G}\left(\bar{T}_{e}-\bar{T}_{p h}\right)=\bar{G} \cdot \bar{T}_{d} \\
= & {\left[2 \times 10^{16}, 2.5 \times 10^{16}\right] . } \\
& \left([1186.7,1171.1]-{ }_{\mathbf{D}}[359.1,372.1]\right) \\
= & {\left[2 \times 10^{16}, 2.5 \times 10^{16}\right] \cdot } \\
& \quad[1186.7-359.1,1171.1-372.1] \\
= & {\left[2 \times 10^{16}, 2.5 \times 10^{16}\right] \cdot[827.6,799] }
\end{aligned}
$$

Taking into account the assumption that $\bar{G}, \bar{T}_{d} \in \mathbf{D} \backslash \mathbf{Z}$ one obtains the following formula

$$
\bar{G} \cdot \bar{T}_{d}=\left[G^{-\sigma\left(\bar{T}_{d}\right)} \cdot T_{d}^{-\sigma(\bar{G})}, G^{\sigma\left(\bar{T}_{d}\right)} \cdot T_{d}^{\sigma(\bar{G})}\right],
$$

For the interval values $\bar{G}=\left[2 \times 10^{16}, 2.5 \times 10^{16}\right]$ and $\bar{T}_{d}=[827.6$, 799] the sign variables are $\sigma(\bar{G})=+, \sigma\left(\bar{T}_{d}\right)=+$, so the product of $\bar{G}$ and $\bar{T}_{d}$ can be calculated according to the formula

$$
\bar{G} \cdot \bar{T}_{d}=\left[G^{-+} \cdot T_{d}^{-+}, G^{+} \cdot T_{d}^{+}\right]=\left[G^{-} \cdot T_{d}^{-}, G^{+} \cdot T_{d}^{+}\right]
$$

and the directed interval phonon energy source is computed as follows 


$$
\begin{aligned}
\bar{Q}_{p h} & =\left[2 \times 10^{16}, 2.5 \times 10^{16}\right] \cdot[827.6,799] \\
& =\left[2 \times 10^{16} \cdot 827.6,2.5 \times 10^{16} \cdot 799\right] . \\
& \approx\left[1.65 \times 10^{19}, 2 \times 10^{19}\right] \mathrm{W} / \mathrm{m}^{3} \mathrm{~K}
\end{aligned}
$$

As a result, the interval obtained is proper.

For one-dimensional model the discrete set of propagation velocities in two lattice directions ( 1 and 2 ) for electrons and phonons is defined as (see Fig. 1)

$$
\begin{gathered}
\mathbf{c}_{e 1}=\left[c_{e}, 0\right] \quad \mathbf{c}_{e 2}=\left[-c_{e}, 0\right] . \\
\mathbf{c}_{p h 1}=\left[c_{p h}, 0\right] \quad \mathbf{c}_{p h 2}=\left[-c_{p h}, 0\right],
\end{gathered}
$$

where $c_{e}=\Delta x_{e} / \Delta t, c_{p h}=\Delta x_{p h} / \Delta t$ are the components of velocity along the $x$-axis for electrons and phonons respectively, $\Delta x_{e}$, $\Delta x_{p h}$ are the lattice distances from site to site, $\Delta t=t^{f+1}-t^{f}$ is the time step needed for a phonon and electron to travel from one lattice site to the neighboring lattice site. In the paper the equality of $\Delta x_{e}$ and $\Delta x_{p h}$ is assumed.

In the LBM method, it is necessary to set the grid in such a way that the result is independent of the grid size. Generally, this can be achieved with a lattice spacing shorter than the phonon mean free path.

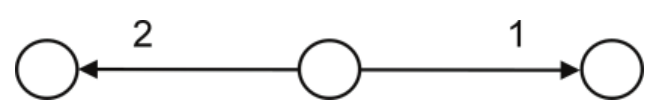

Fig. 1. One-dimensional 2-speed lattice Boltzmann model

The equations (22) and (23) should be supplemented by the boundary conditions [28], for example of the $2^{\text {nd }}$ type on the left boundary and the $1^{\text {st }}$ type on the right boundary as follows

$$
\begin{cases}x=0: & \bar{q}_{b}^{e}(0, t)=c_{e}\left[\bar{e}_{e}\left(\bar{T}_{e}\right)_{0}-\bar{e}_{e}\left(\bar{T}_{e}\right)_{1}\right] \\ x=L: & e_{e}(L, t)=e_{e}\left(T_{b 2}^{e}\right) \\ x=0: & \bar{q}_{b}^{p h}(0, t)=c_{p h}\left[\bar{e}_{p h}\left(\bar{T}_{p h}\right)_{0}-\bar{e}_{p h}\left(\bar{T}_{p h}\right)_{1}\right] \\ x=L: & e_{p h}(L, t)=e_{p h}\left(T_{b 2}^{p h}\right)\end{cases}
$$

and the initial conditions

$$
\begin{array}{ll}
t=0: & e_{e}(x, 0)=e_{e}\left(T_{0}^{e}\right) \\
t=0: & e_{p h}(x, 0)=e_{p h}\left(T_{0}^{p h}\right)
\end{array}
$$

where $T_{b 2}^{e}$ and $T_{b 2}^{p h}$ are boundary temperatures of electrons and phonons respectively, $\left(\bar{T}_{e}\right)_{0}$ and $\left(\bar{T}_{e}\right)_{1}$ are temperatures of electrons in the first and second node, $\left(\bar{T}_{p h}\right)_{0}$ and $\left(\bar{T}_{p h}\right)_{1}$ are temperatures of phonons in the first and second node, $T_{0}^{e}$ is the electron's initial temperature and $T_{0}^{p h}$ is the phonon's initial temperature. Taking into account equations $(30,31)$, the set of four interval partial differential equations is obtained

$$
\left\{\begin{array}{l}
\frac{\partial \bar{e}_{e 1}}{\partial t}+c_{e} \frac{\partial \bar{e}_{e 1}}{\partial x}=-\frac{\bar{e}_{e 1}-\bar{e}_{e 1}{ }^{0}}{\bar{\tau}_{e}}+\bar{Q}_{e} \\
\frac{\partial \bar{e}_{e 2}}{\partial t}-c_{e} \frac{\partial \bar{e}_{e 2}}{\partial x}=-\frac{\bar{e}_{e 2}-\bar{e}_{e 2}{ }^{0}}{\bar{\tau}_{e}}+\bar{Q}_{e} \\
\frac{\partial \bar{e}_{p h 1}}{\partial t}+c_{p h} \frac{\partial \bar{e}_{p h 1}}{\partial x}=-\frac{\bar{e}_{p h 1}-\bar{e}_{p h 1}{ }^{0}}{\bar{\tau}_{p h}}+\bar{Q}_{p h} \\
\frac{\partial \bar{e}_{p h 2}}{\partial t}-c_{p h} \frac{\partial \bar{e}_{p h 2}}{\partial x}=-\frac{\bar{e}_{p h 2}-\bar{e}_{p h 2}{ }^{0}}{\bar{\tau}_{p h}}+\bar{Q}_{p h}
\end{array} .\right.
$$

Introducing discretizing form, time and position derivatives may be written as follows

$$
\left\{\begin{array}{l}
\left(\bar{e}_{e 1}\right)_{i+1}^{f+1}=\left(1-\frac{\Delta t}{\bar{\tau}_{e}}\right)\left(\bar{e}_{e 1}\right)_{i}^{f}+\frac{\Delta t}{\bar{\tau}_{e}}\left(\bar{e}_{e 1}^{0}\right)_{i}^{f}+\Delta t \bar{Q}_{e} \\
\left(\bar{e}_{e 2}\right)_{i-1}^{f+1}=\left(1-\frac{\Delta t}{\bar{\tau}_{e}}\right)\left(\bar{e}_{e 2}\right)_{i}^{f}+\frac{\Delta t}{\bar{\tau}_{e}}\left(\bar{e}_{e 2}^{0}\right)_{i}^{f}+\Delta t \bar{Q}_{e} \\
\left(\bar{e}_{p h 1}\right)_{i+1}^{f+1}=\left(1-\frac{\Delta t}{\bar{\tau}_{p h}}\right)\left(\bar{e}_{p h 1}\right)_{i}^{f}+\frac{\Delta t}{\bar{\tau}_{p h}}\left(\bar{e}_{p h 1}^{0}\right)_{i}^{f}+\Delta t \bar{Q}_{p h} \\
\left(\bar{e}_{p h 2}\right)_{i-1}^{f+1}=\left(1-\frac{\Delta t}{\bar{\tau}_{p h}}\right)\left(\bar{e}_{p h 2}\right)_{i}^{f}+\frac{\Delta t}{\bar{\tau}_{p h}}\left(\bar{e}_{p h 2}^{0}\right)_{i}^{f}+\Delta t \bar{Q}_{p h}
\end{array} .\right.
$$

For example, for the interval phonon relaxation time $\bar{\tau}_{p h}=[0.76,0.84] \mathrm{ps}$ and the time step $\Delta t=0.01 \mathrm{ps}$, the value of the quotient $\Delta t / \bar{\tau}_{p h}$ is calculated according to the formula (see (13))

$$
\Delta t / \bar{\tau}_{p h}=\bar{\Delta} t / \bar{\tau}_{p h}=[0.01,0.01] /_{\mathbf{D}}[0.76,0.84] .
$$

Taking into account the assumption that $\bar{\Delta} t, \bar{\tau}_{p h} \in \mathbf{D} \backslash \mathbf{Z}$ one obtains the following formula

$$
\bar{\Delta} t / \bar{\tau}_{p h}=\left[\Delta t^{-\sigma\left(\bar{\tau}_{p h}\right)} / \tau_{p h}{ }^{-\sigma(\bar{\Delta} t)}, \Delta t^{\sigma\left(\bar{\tau}_{p h}\right)} / \tau_{p h}{ }^{\sigma(\bar{\Delta} t)}\right] .
$$

For the interval values $\bar{\Delta} t=[0.01,0.01] \mathrm{ps}$ and $\bar{\tau}_{p h}=[0.76,0.84] \mathrm{ps}$ the sign variables are $\sigma(\bar{\Delta} t)=+, \sigma\left(\bar{\tau}_{p h}\right)=+$ so the quotient $\Delta t / \bar{\tau}_{p h}$ can be calculated according to the formula

$$
\begin{aligned}
\bar{\Delta} t / \bar{\tau}_{p h} & =\left[\Delta t^{-+} / \tau_{p h}{ }^{-+}, \Delta t^{+} / \tau_{p h}{ }^{+}\right] \\
& =\left[\Delta t^{-} / \tau_{p h}{ }^{-}, \Delta t^{+} / \tau_{p h}^{+}\right]
\end{aligned}
$$

and finally the quotient is computed as follows

$$
\bar{\Delta} t / \bar{\tau}_{p h}=[0.01 / 0.76,0.01 / 0.84] \approx[0.013,0.012] \text {. }
$$

As a result, the interval obtained is improper.

The total energy density for electrons and phonons is defined as the sum of discrete energy densities in all the lattice directions and takes the form 


$$
\begin{aligned}
& \left(\bar{e}_{e}\right)_{i}^{f}=\left(\bar{e}_{e 1}\right)_{i}^{f}+\left(\bar{e}_{e 2}\right)_{i}^{f} \\
& \left(\bar{e}_{p h}\right)_{i}^{f}=\left(\bar{e}_{p h 1}\right)_{i}^{f}+\left(\bar{e}_{p h 2}\right)_{i}^{f} .
\end{aligned}
$$

The equilibrium electron energy density and phonon energy density is the same in all lattice directions and can be calculated using the formula [32]:

$$
\begin{gathered}
\left(\bar{e}_{e}^{0}\right)_{i}^{f}=\frac{\left(\bar{e}_{e}\right)_{i}^{f}}{2}, \\
\left(\bar{e}_{p h}^{0}\right)_{i}^{f}=\frac{\left(\bar{e}_{p h}\right)_{i}^{f}}{2} .
\end{gathered}
$$

After subsequent computations the lattice temperature is determined using the formula

$$
\begin{aligned}
& \left(\bar{T}_{e}\right)_{i}^{f+1}=\sqrt{\bar{e}_{e}\left[\left(\bar{T}_{e}\right)_{i}^{f}\right] /\left(n_{e} \frac{\pi^{2}}{2} \frac{k_{b}^{2}}{\varepsilon_{F}}\right)} \\
& \left(\bar{T}_{p h}\right)_{i}^{f+1}=\sqrt[4]{\frac{\bar{e}\left[\left(\bar{T}_{p h}\right)_{i}^{f}\right] \Theta_{D}^{3}}{9 \eta k_{b} \int_{0}^{\Theta_{D} / \bar{T}_{p h}^{f}} \frac{z^{3}}{\exp (z)-1} \mathrm{~d} z}} .
\end{aligned}
$$

\section{Results of computations}

As a numerical example the heat transport in a gold thin film of thickness $200 \mathrm{~nm}$ has been analyzed. The most common values of phonon relaxation time is $0.8 \mathrm{ps}$ and electron relaxation time $0.04 \mathrm{ps}$ [33], while the value of the electron-phonon coupling factor is $2.3 \times 10^{16} \mathrm{~W} / \mathrm{m}^{3} \mathrm{~K}$ [12]. In numerical examples the following input data have been introduced: the relaxation time $\bar{\tau}_{e}=[0.038,0.042] \mathrm{ps}, \bar{\tau}_{p h}=[0.76,0.84] \mathrm{ps}$ the Debye temperature $\Theta_{D}=170 \mathrm{~K}$, the internal source function $Q^{\prime}=10^{20} \mathrm{~W} / \mathrm{m}^{3}$,

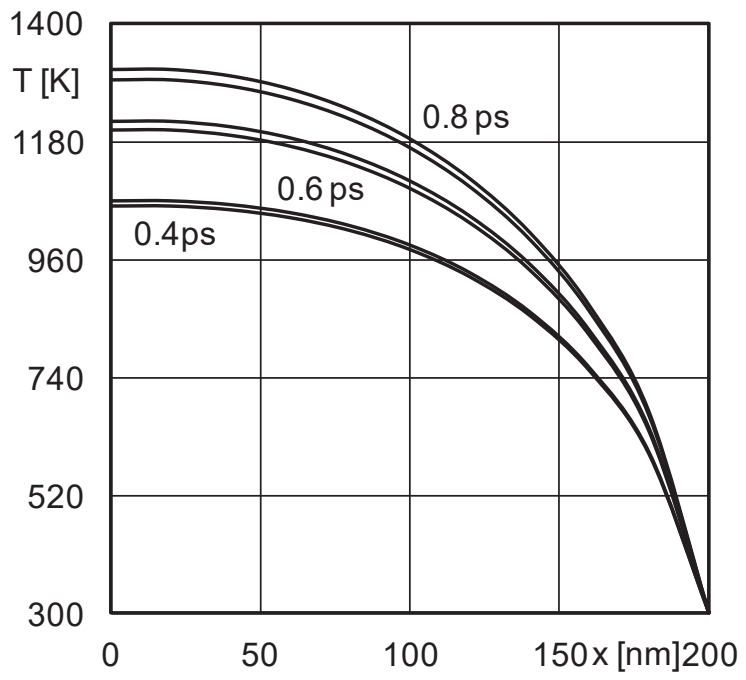

Fig. 2. The interval temperature distribution of electrons in a gold film the interval coupling factor $\bar{G}=\left[2 \times 10^{16}, 2.5 \times 10^{16}\right] \mathrm{W} / \mathrm{m}^{3} \mathrm{~K}$ in the first variant and $\bar{G}=\left[1.8 \times 10^{16}, 2.7 \times 10^{16}\right] \mathrm{W} / \mathrm{m}^{3} \mathrm{~K}$ in the second variant, the boundary conditions of the $2^{\text {nd }}$ type on the left boundary $\bar{q}_{b}^{e}(0, t)=\bar{q}_{b}^{p h}(0, t)=\overline{0}$ and the $1^{\text {st }}$ type on the right boundary $T_{b 2}^{e}=T_{b 2}^{p h}=300 \mathrm{~K}$, the initial temperature $T_{0}=300 \mathrm{~K}$. The lattice step $\Delta x=20 \mathrm{~nm}$ and the time step $\Delta t=0.01 \mathrm{ps}$ have been assumed.

Figure 2 illustrates the interval temperature distribution of electrons in the domain considered for the chosen times, while in Fig. 3 the interval temperature distribution of electrons for wider interval of the coupling factor for the same chosen times is shown.

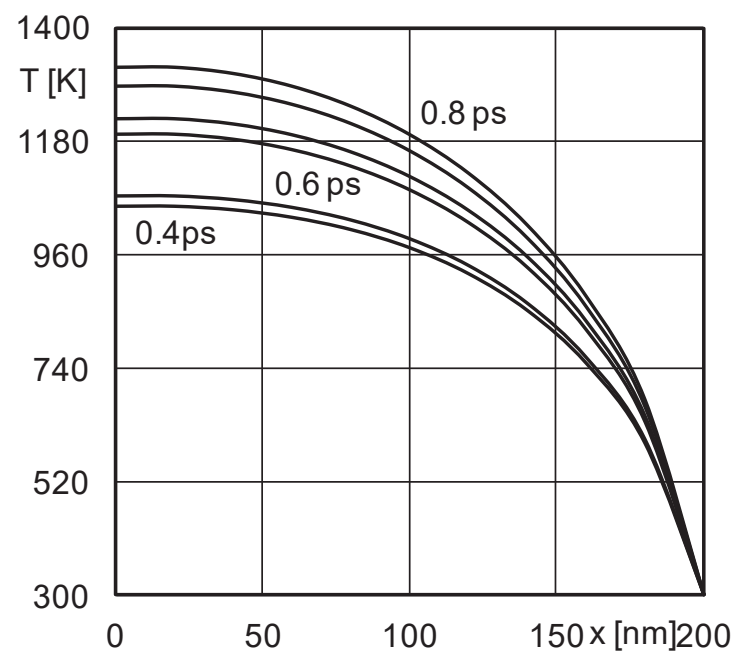

Fig. 3. The interval temperature distribution of electrons in a gold film for wider interval of the coupling factor

Figure 4 presents the interval temperature distribution of phonons in the domain considered for the chosen times.

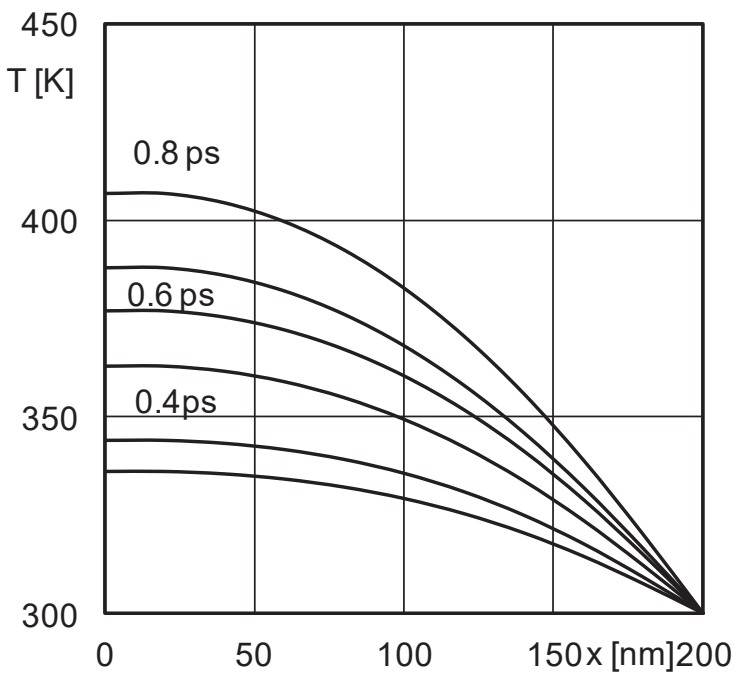

Fig. 4. The interval temperature distribution of phonons in a gold film 


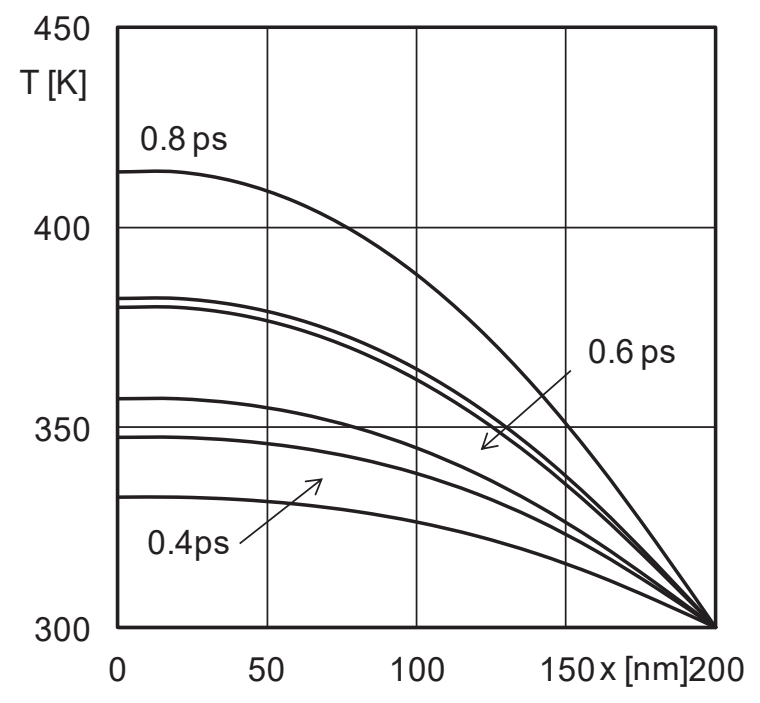

Fig. 5. The interval temperature distribution of phonons in a gold film for wider interval of the coupling factor

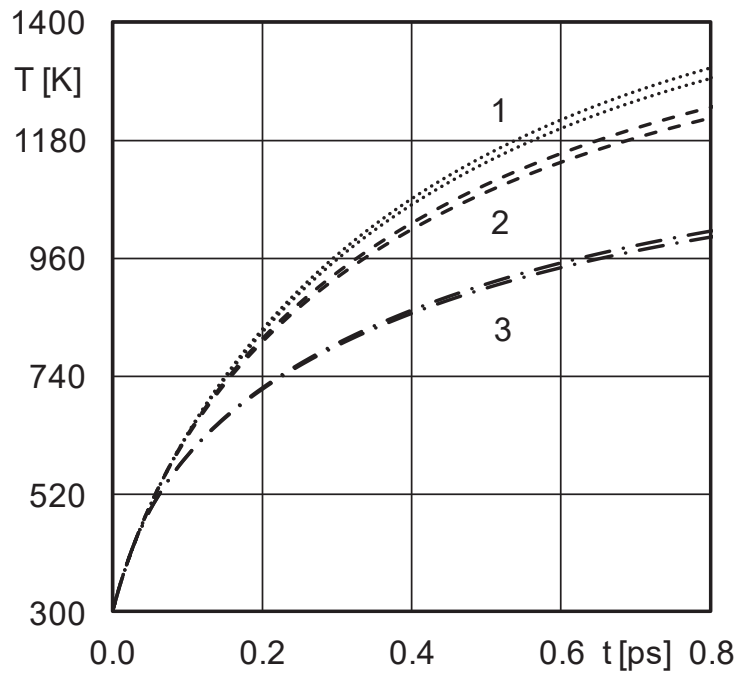

Fig. 6. Heating curves at internal nodes (temperature of electrons)

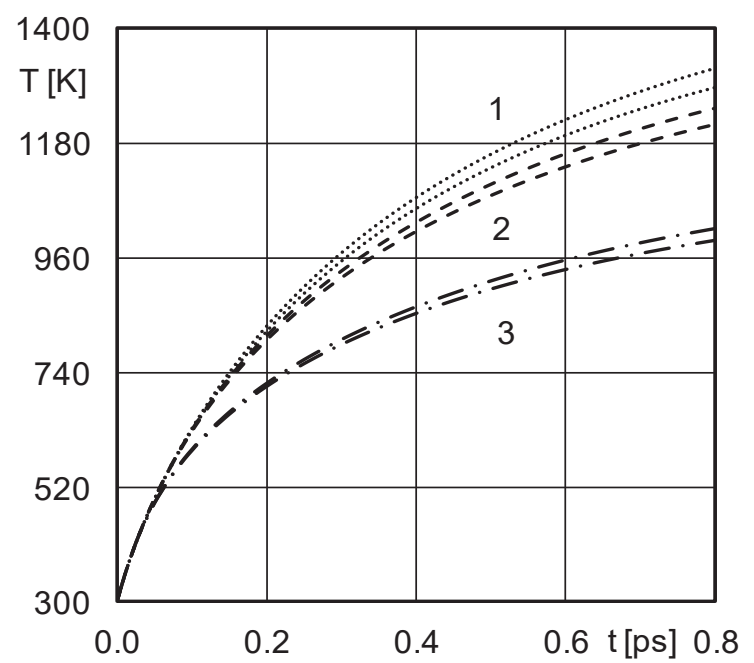

Fig. 7. Heating curves at internal nodes for wider interval of the coupling factor (temperature of electrons)
Figure 5 illustrates the interval temperature distribution of phonons for wider interval of the coupling factor for the same chosen times.

In Fig. 6 the courses of the temperature of electrons at the internal nodes $1(20 \mathrm{~nm}), 2(80 \mathrm{~nm})$ and $3(140 \mathrm{~nm})$ are shown.

Figure 7 illustrates the interval heating curves at the same internal nodes for wider interval of the coupling factor and then the interval temperatures are wider, of course.

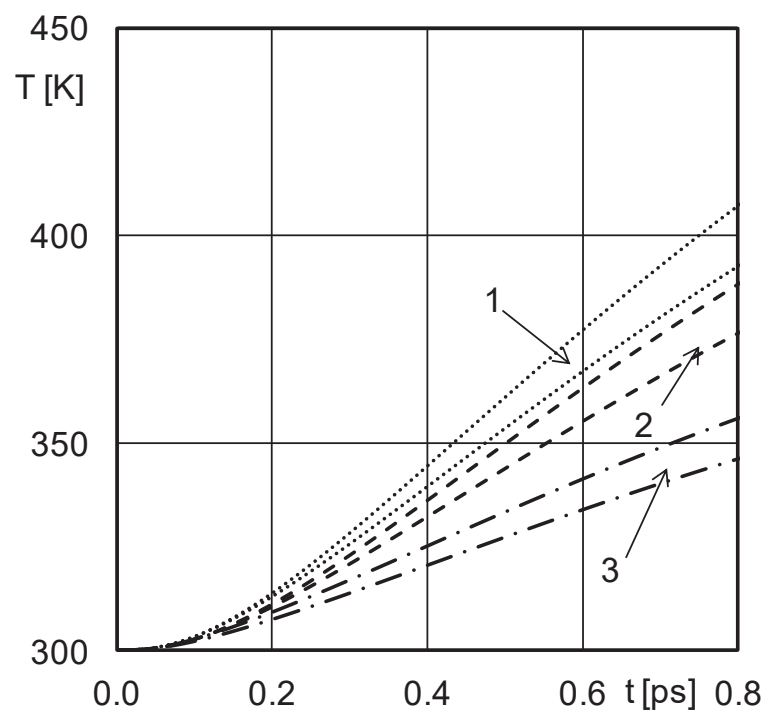

Fig. 8. Heating curves at internal nodes (temperature of phonons)

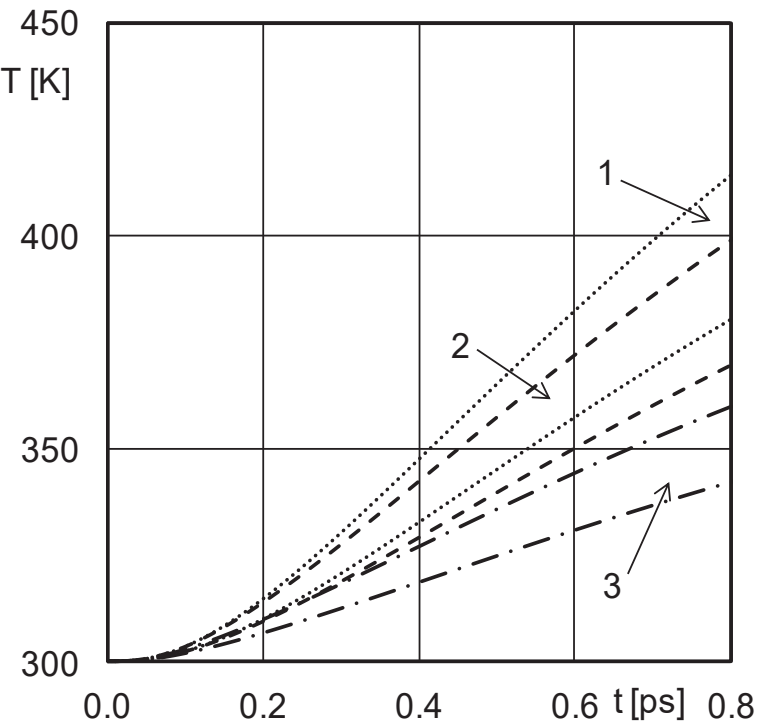

Fig. 9. Heating curves at internal nodes for wider interval of the coupling factor (temperature of phonons)

Figures 8 and 9 show the phonon interval heating curves at the same internal nodes for narrow and wider interval of the coupling factor, respectively. 


\section{Conclusions}

In the paper the coupled lattice Boltzmann equations for electrons and phonons are applied to analyze the heating process of the thin metal films via laser pulse. The Boltzmann transport equations with the interval values of the relaxation time and the phonon-electron coupling factor have been considered. The interval version of the lattice Boltzmann method for solving 1D problems has been presented using the rules of directed interval arithmetic. The generalization of LBM allows one to find the numerical solution in the interval form and such an information may be important especially for the parameters which are estimated experimentally, for example the relaxation times. The main advantage of the directed interval arithmetic upon the classical interval arithmetic is that the obtained temperature intervals are much narrower. It should be pointed out that the method proposed can be extended to multi-layered domains $[34,35]$.

\section{REFERENCES}

[1] M. Eshraghi, S.D. Felicelli, “An implicit lattice Boltzmann model for heat conduction with chase chandler", Int. J. of Heat and Mass Transfer 55, 2420-2428 (2012).

[2] R.A. Escobar, C.H. Amon, "Thin film phonon heat conduction by the dispersion lattice Boltzmann method", J. Heat Transfer 130(9), 092402 1-8 (2008).

[3] G.R. McNamara, G. Zanetti, "Use of the Boltzmann equation to simulate lattice-gas automata", Phys. Rev. Lett. 61 (20), 2332-2335 (1988).

[4] F. Higuera, S. Succi, R. Benzi, "Lattice gas dynamics with enhanced collisions", Europhys. Let. 9 (4), 345-349 (1989).

[5] R. Benzi, S. Succi, M. Vergassola, "The lattice Boltzmann equation: theory and applications", Phys. Rep. 222 (3), 145-197 (1992).

[6] E. Majchrzak, G. Kałuża, "Heat flux formulation for 1D dual-phase lag equation", J. of Applied Mathematics and Computational Mechanics 14(1), 71-78 (2015).

[7] E. Majchrzak, B. Mochnacki, "Sensitivity analysis of transient temperature field in microdomains with respect to the dual phase lag model parameters", Int. J. for Multiscale Computational Engineering12(1), 65-77 (2014).

[8] E. Majchrzak, J. Dziatkiewicz, "Analysis of ultashort laser pulse interactions with metal films using a two-temperature model", J. of Applied Mathematics and Computational Mechanics 14(2), 31-39 (2015).

[9] A. Maroufi, C. Aghanajafi, "Analysis of conduction-radiation heat transfer during phase change process of semitransparent materials using lattice Boltzmann method", $J$. of Quantitative Spectroscopy \& Radiative Transfer 116, 145-155 (2013).

[10] S.C. Mishra, B. Mondal, T. Kush, B.S.R. Krishna, "Solving transient heat conduction problems on uniform and non-uniform lattices using the lattice Boltzmann method", Int. Communications in Heat and Mass Transfer 36, 322-328 (2009).

[11] D. Wang, Z. Qu, Y. Ma, "An enhanced Gray model for nondiffusive heat conduction solved by implicit lattice Boltzmann method", J. of Heat and Mass Transfer 94, 411-418 (2016).

[12] P.E. Hopkins, P.M. Norris, "Contribution of ballistic electron transport to energy transfer during electron-phonon nonequi in thin metal films", J. Heat Transfer, 131(4), 043208 1-8 (2009).
[13] J.K. Chen, D.Y. Tzou, J.E. Beraun, "A semiclassical two-temperature model for ultrafast laser heating", Int. J. of Heat and Mass Transfer 49, 307-316 (2006).

[14] A.A. Joshi, A. Majumdar, "Transient ballistic and diffusive phonon heat transport in thin films", J. of Appl. Physics 74(1), 31-39 (1993).

[15] A. Piasecka Belkhayat, A. Korczak, Modelling of Transient Heat Transport in One-Dimensional Crystalline Solids Using the Interval Lattice Boltzmann Method, 363-368 Taylor \& Francis Group, London (2014).

[16] A. Piasecka Belkhayat, A. Korczak, "Numerical modelling of the transient heat transport in $2 \mathrm{~d}$ silicon thin film using the interval lattice Boltzmann method", J. of Appl. Mathematics and Comp. Mechanics 13 (2), 95-103 (2014).

[17] A. Piasecka Belkhayat, "The interval lattice Boltzmann method for transient heat transport", Scientific Research of the Institute of Mathematics and Computer Science 1(8), 155-160 (2009).

[18] M. Kaviany, A.J.H. McGaughey, J.D. Chung, "Integration of molecular dynamics simulations and Boltzmann transport equation in phonon thermal conductivity analysis", Heat Transfer 4, 277-287 (2003).

[19] J.B. Lee, K. Kang, S.H. Lee, “Comparison of theoretical models of electron-phonon coupling in thin gold films irradiated by femtosecond pulse lasers", Materials Transactions 52 (3), 547-553 (2011).

[20] S.M. Markov, "On directed interval arithmetic and its applications", J. of Universal Comp. Science 1, 514-526 (1995).

[21] A. Piasecka Belkhayat, A. Korczak, "Numerical modelling of the transient heat transport in a thin gold film using the fuzzy lattice Boltzmann method with $\alpha$-cuts", J. of Applied Mathematics and Computational Mechanics 15(1), 99-111 (2016).

[22] B. Mochnacki, A. Piasecka Belkhayat, "Numerical modeling of skin tissue heating using the interval finite difference method", Molecular \& Cellular Biomechanics 10 (3), 233-244 (2013).

[23] A. Piasecka Belkhayat, "Interval boundary element method for 2D transient diffusion problem", Engineering Analysis with Boundary Elements 32 (5), 424-430 (2008).

[24] A. Piasecka Belkhayat, Interval Boundary Element Method for Imprecisely Defined Unsteady Heat Transfer Problems, Monograph (321), Silesian University of Technology, Gliwice, 2011, (in Polish).

[25] A. Neumaier, Interval Methods for System of Equations, Cambridge University Press, Cambridge, New York, Port Chester, Melbourne, Sydney, 1990.

[26] S.S. Ghai, W.T. Kim, R.A. Escobar, et al., "A novel heat transfer model and its application to information storage systems", J. Appl. Physics 97, 10P703 1-3 (2005).

[27] S. Pisipati, J. Geer, B. Sammakia, B.T. Murray, "A novel alternate approach for multiscale thermal transport using diffusion in the Boltzmann Transport Equation", Int. J. of Heat and Mass Transfer 54, 3406-3419 (2011).

[28] R.A. Escobar, S.S. Ghai, M.S. Jhon, C.H. Amon, "Multi-length and time scale thermal transport using the lattice Boltzmann method with application to electronics cooling", J. of Heat and Mass Transfer 49, 97-107 (2006).

[29] K. Venkatakrishnan, B. Tan, B.K.A. Ngoi, "Femtosecond pulsed laser ablation of thin gold film", Optics \& Laser Technology 34, 199 - 202 (2002).

[30] X. Zhang, S.S. Chu, J.R. Ho, Grigoropoulos C.P., "Excimer laser ablation of thin gold films on a quartz crystal microbalance at various argon background pressures", Appl. Physics A 64, 545-552 (1997). 
[31] B.S. Yilbas, A.Y. Al-Dweik, Bin Mansour S., "Analytical solution of hyperbolic heat conduction equation in relation to laser short-pulse heating", Physica B 406, 1550-1556 (2011).

[32] R. Escobar, B. Smith, C. Amon, "Lattice Boltzmann modeling of subcontinuum energy transport in crystalline and amorphous microelectronic devices", J. of Electronic Packaging 128 (2), 115-124 (2006).

[33] J.K. Chen, J.E. Beraun, "Numerical study of ultrashort laser pulse interactions with metal films", Numerical Heat Transfer, Part A, 40, pp. 1-20, 2001
[34] E. Majchrzak, B. Mochnacki, A.L. Greer, J.S. Suchy, "Numerical modeling of short pulse laser interactions with multi-layered thin metal films", CMES: Computer Modeling in Engineering and Sciences, 41 (2), 131-146 (2009).

[35] E. Majchrzak, B. Mochnacki, J.S. Suchy, "Numerical simulation of thermal processes proceeding in a multi-layered film subjected to ultrafast laser heating", J. of Theor. and Appl. Mechanics 47 (2), 383-396 (2009). 